\title{
EDAC: A Novel Energy-Aware Clustering Algorithm for Wireless Sensor Networks
}

\author{
Ahmad A. Ababneh \\ Electrical Engineering Department \\ Jordan University of Science \& Technology \\ Irbid, Jordan
}

\author{
Ebtessam Al-Zboun \\ Electrical Engineering Department \\ Jordan University of Science \& Technology \\ Irbid, Jordan
}

\begin{abstract}
Clustering is a useful technique for reducing energy consumption in wireless sensor networks (WSN). To achieve a better network lifetime performance, different clustering algorithms use various parameters for cluster head (CH) selection. For example, the sensor's own residual energy as well as the network's total residual energy are used. In this paper, we propose an energy-distance aware clustering (EDAC) algorithm that incorporates both the residual energy levels of sensors within a cluster radius as well as the distances. To achieve this, we define a metric that is calculated at each sensor based on local information within its neighborhood. This metric is incorporated within the $\mathrm{CH}$ selection probability. Using this metric, one can choose the sensors with low residual energy levels to have the greatest impact on $\mathrm{CH}$ selection which results in $\mathrm{CH}$ selection being biased to be close to these sensors. This results in reducing their communication energy cost to the $\mathrm{CH}$. Simulation results indicate that our proposed EDAC algorithm outperforms both the LEACH and the energy-efficient DEEC protocols in terms of network lifetime.
\end{abstract}

\section{Keywords-Clustering algorithms; Sensor networks}

\section{INTRODUCTION}

Wireless sensor networks (WSNs) have garnered much attention in the last decade. This is as a result of advances in networking, wireless communication, micro-fabrications, micro-processors, and the wide range of applications [1]. WSN consists of a number of sensor nodes deployed in an area of interest. Sensors collect data and send data to a central processor (e.g. Base Station (BS)). WSNs have important applications such as remote environmental monitoring [3], military applications (e.g., battlefield surveillance) [2], and industrial monitoring applications which include machine health monitoring, and industrial control applications[4]. One of the challenges faces the use of WSN is energy efficiency because it can be difficult (e.g., hazardous areas) to replace the batteries, so the design of energy- efficient network protocols becomes an important issue with respect to network lifetime extension [5]. A major source of energy dissipation is communication between sensors and the base station. To guarantee a good balanced distribution of the energy load between sensor nodes, clustering communication protocols have been designed and implemented.

In WSN, the clustering protocol is a key factor in achieving energy efficiency, so the design of an energy-efficient clustering protocol for WSN is very important. In WSNs the sensor nodes are energy constrained. Therefore, it is very important to find some solutions to offer high scalability and satisfy high energy efficiency to prolong network lifetime. One solution solution is by grouping sensor nodes into sets called clusters. Clustering achieves better lifetime of the sensor network by breaking the sensor network into groups of sensors to conserve communication energy. As a result, saving the energy and increasing the overall lifetime of the network is achieved.

Adopting clustering scheme produces two-level hierarchy; the higher level and the lower level. The higher level is formed by the nodes that are responsible for aggregating and fusing the received data from sensor nodes in the sensing area and then transmit it to a central processor; such nodes are called the Cluster Head $(\mathrm{CH})$ nodes. The lower level of the hierarchy is formed by the nodes that are responsible for detecting the required data from the sensing region and then sending it to the corresponding $\mathrm{CH}$. Each cluster includes number of sensor nodes and one cluster head $(\mathrm{CH})$ [6]. $\mathrm{CH}$ selection can be centralized performed by the BS or the end user based on some criterion. It can also be distributed in nature and performed by the sensors themselves on a localized level. The BS is responsible for processing data received from sensor nodes to be used by the end user.

In this paper, we propose a novel distributed energyefficient cluster head selection algorithm in which two factors are incorporated: the sensors' residual energy levels and the distances between sensors and the $\mathrm{CH}$.

The rest of the paper is arranged as follows. In section 2, a literature review about several clustering algorithms is introduced. In section 3, the network model and the energy expenditure model are adopted. In section 4, we show how the proposed protocol will be used in the process of cluster head selection, and simulation results are explained in section 5 .

\section{LITERATURE REVIEW}

The most important and widely used probabilistic clustering protocols are LEACH [7], HEED [8] and DEEC [9]. In $\mathrm{LEACH}$, the $\mathrm{CH}$ is selected using rotation. The selection of cluster heads is based on setting a predefined percentage of $\mathrm{CHs}$ for the network. In the LEACH algorithm, each sensor locally calculates a random number and compares it to some threshold that depends on the percentage of $\mathrm{CH}$ needed. LEACH performance in homogeneous network (i.e., of the same energy level) is good, whereas in heterogeneous network it is not. HEED is a hierarchical, distributed, clustering algorithm, this algorithm uses both of the remaining energy of 
the sensor node and the intra-cluster communication periodically in the scheme of cluster head selection. Another algorithm is DEEC which is tailored for use in heterogeneous networks. DEEC uses a probability that is implemented as a ratio between the sensor remaining energy and the network average energy. Nodes with high initial and residual energy will be elected to be $\mathrm{CHs}$ with higher probability than that with low initial and residual energy in the network. In [10], SEP is proposed for the two-level heterogeneous networks, where the two-level heterogeneous network includes two types of sensor nodes; the normal nodes and the advanced nodes, in this protocol the process of cluster head selection consists of rounds, the decision of being a cluster head or not is made by the sensor node at each round based on its initial energy relative to that of other nodes.

\section{SySTEM MODEL AND ENERGY EXPEDITURE MODEL}

In this section, we will introduce the system model and then the energy expenditure model will be explained.

\section{A. System model}

For simplicity, we model the sensing area as a 2-D square area of dimension $M$. A group of $N$ static sensor nodes is dispersed randomly as shown in Figure (1), [7]. The network is divided into clusters, and the CHs transmit the fused and the aggregated data to the BS which is located at the center of the sensing region.

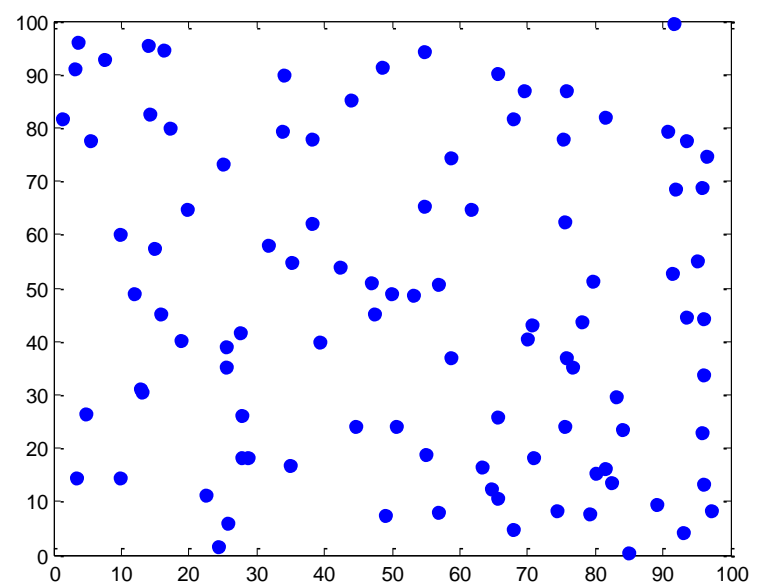

Fig. 1. 100-Nodes Random Networks

For the two-level heterogeneous networks, the sensor nodes are classified into advanced nodes and normal nodes, in which two different levels of energy are assigned. Let $m$ denote the fraction of advanced nodes in the network with initial energy $E_{i}$, and is provided with $a$ times excessive energy compared to the normal nodes then the network will contain $m N$ advanced nodes that are supplied with $E_{i}(1+a)$ initial energy, and $(1-m) N$ normal nodes supplied with $E_{i}$ initial energy. Thus, the total initial energy of the network in this case can be obtained as:

$$
E_{\text {tot }}=N(1-m) E_{\text {ini }}+N m(1+a) E_{\text {ini }}=N E_{\text {ini }}(1+a m)
$$

On the other hand, it is possible to equip the sensor nodes with multi-level of energy; in this case, the advanced node $s_{i}$ is provided with initial energy of $E_{i}\left(1+a_{i}\right)$ which is $a_{i}$ times more energy compared to the initial energy of the normal nodes. We note that $a_{i}$ can be a random quantity. Thus, the total initial energy of the network can be written as:

$$
E_{\text {tot }}=\sum_{i=1}^{N} E_{i}\left(1+a_{i}\right)=E_{i}\left(N+\sum_{i=1}^{N} a_{i}\right)
$$

The following assumptions are held:

- Sensor nodes are aware of their locations.

- Communication channel is symmetric, between $\mathrm{CH}$ and corresponding sensors

- Single hop communication between sensors and their $\mathrm{CH}$.

\section{B. Energy Expenditure Model}

Communication between sensor nodes dissipates most of its energy depending on the distance between the sending and receiving sensor nodes. We use the 1st order radio frequency energy consumption model to describe the energy consumption for sensor nodes [11] which incorporates both free-space and multi-path energy loss. According to this model, shown in Figure (2) [7,12], the energy spent for transmitting $l$-bit data message to a sensor at distance $d$ is given as

$$
E_{T X}(l, d)= \begin{cases}l E_{\text {elec }}+\epsilon_{f s} d^{2} & d<d_{0} \\ l E_{\text {elec }}+\epsilon_{m p} d^{4} & d>d_{0}\end{cases}
$$

Where $l E_{\text {elec }}$ is the electronic energy which is the energy dissipated to operate the transmitter or the receiver circuit to process one bit, $\epsilon_{f s}$ and $\epsilon_{m p}$ are the amplifier parameters of transmission corresponding to the free-space model and multipath respectively, $\quad d_{0=} \sqrt{\epsilon_{f s} / \epsilon_{m p}}$ denotes the threshold distance, and $d$ is the distance between sensor node $s_{i}$ and sensor node $s_{i}$ and given as:

$$
\left|d_{\{i, j\}}\right|=\sqrt{\left(x_{i}-x_{j}\right)^{2}+\left(y_{i}-y_{j}\right)^{2}}
$$

Similarly, the energy consumed to receive this message is given as

$$
E_{R X}(l)=l \times E_{\text {elec }}
$$

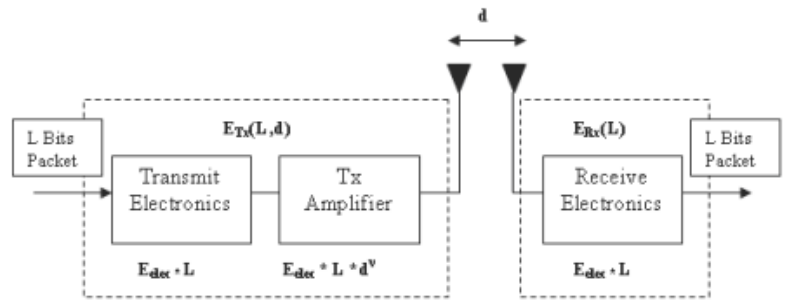

Fig. 2. Radio Energy Dissipation Model

\section{Proposed Clustering Protocol}

In this section, we will introduce our proposed clustering protocol called the EDAC. The goal of this protocol is to incorporate the residual energy of sensor node with the cluster head selection process. In contrast to other clustering protocols that only incorporate the residual energy of sensor nodes, we 
also incorporate on energy distance measure in the selection process.

We first define our distance-energy metric and then explain the steps of our protocol.

\section{A. Distance-Energy Metric}

It is necessary to propose a metric that quantifies how good a sensor node could be as a $\mathrm{CH}$. This metric needs to take into account both the residual energy of sensor nodes in addition to the energy expenditure in transmitting data in intra-cluster communication. As noted from energy model, the energy expended in intra-cluster communication is proportional with distance, therefore it is preferred for a $\mathrm{CH}$ to be as close as possible to the sensor nodes in its cluster radius. Moreover, sensor nodes with a low residual energy should have more impact in the $\mathrm{CH}$ selection process.

The sensor nodes residual energy can be calculated during a single frame/epoch as

$$
E_{\text {res }(i)}=E_{i}-E_{\operatorname{diss}(i)}
$$

Where $E_{i}$ is the sensor node energy for the current round, and $E_{\text {diss }(i)}$ is the energy dissipated in the $n^{\text {th }}$ sensor node. For simplicity, assume that the maximum distance between any sensor node and the $\mathrm{BS}$ is $<d_{0}$, the $\mathrm{CH}$ dissipated energy will be:

$$
E_{C H}=L E_{\text {elec }}\left(\frac{N}{k}\right)+L E_{D A}\left(\frac{N}{k}\right)+L E_{\text {elec }}+L \epsilon_{f S} d_{\text {to } B S}^{2}
$$

Where $k$ is the number clusters, $E_{D A}$ is the consumed energy when processing a bit per signal and $d_{t o}^{2} B S$ represents the distance from the $\mathrm{CH}$ to the $\mathrm{BS}$. Where the dissipated energy in the non- $\mathrm{CH}$ node is given by the following formula:

$$
E_{\text {non-CH}}=L E_{\text {elec }}+L \epsilon_{f s} d_{\text {to } C H}^{2}
$$

Where $d_{t o C H}^{2}$ represents the distance from the sensor node to its $\mathrm{CH}$. $\mathrm{CH}$ needs energy to receive the sensed data from the sensor nodes within its cluster, to aggregate and to transmit these data to the BS. Sensor node dissipates energy only when transmitting its sensed data to its $\mathrm{CH}$. Thus, the total energy dissipated in the cluster during a round can be obtained as:

$$
E_{\text {Cluster }}=E_{C H}+\left(\frac{N}{k}\right) E_{n o n-C H}
$$

And the total dissipated energy in the network is equal to:

$$
E_{\text {round }}=L\left(2 N E_{\text {elec }}+N E_{D A}+k \epsilon_{m p} d_{\text {to BS }}^{4}+N \epsilon_{f s} d_{\text {to } C H}^{2}\right)
$$

Taking into account the uniform distribution of nodes in the network, the following can be obtained [11], [13]:

$$
d_{t o C H}=\frac{M}{\sqrt{2 \pi k}}, d_{\text {to } B S}=0.765 \frac{M}{2}
$$

Now, the optimal number of cluster heads can be found by differentiating $E_{\text {round }}$ with respect to $k$ and equating to zero.

$$
k_{o p t}=\sqrt{\frac{N}{2 \pi}} \sqrt{\frac{\epsilon_{f s}}{\epsilon_{m p}}} \frac{M}{d_{t o B S}^{2}}
$$

\section{B. The Proposed Protocol(EDAC)}

The main idea behind our proposed protocol is to incorporate not only the sensor's own residual energy but also the residual energy levels of sensors within its clustering radius. Moreover, we incorporate the distance between a sensor and the nearby sensors. The lower the residual energy level of a sensor, the more important it becomes to reduce communication energy by placing the $\mathrm{CH}$ close to it. This will have the effect of reducing overall energy consumption in the network and extending its lifetime.

The first step in the proposed protocol is to calculate the sensor node residual energy and the distance to the sensor nodes, then the $i^{\text {th }}$ sensor node calculates the clustering weight as

$$
w_{s}(i)=\sum_{j \in I_{i}}\left(\alpha R_{-} E_{r e s(j)} d_{i m}+(1-\alpha) \frac{\left(1-R_{-} E_{r e s(j)}\right)}{d_{i j}}\right)
$$

Where, summation is taken over all sensors in the set $I_{i}$ which is the set of sensors within the clustering radius of the $i$ th node and $d_{i j}$ is the distance between sensor node $i$ and sensor node $j$. The quantity $R_{-} E_{\text {res }(j)}$ denotes the relative residual energy of the node and is given as

$$
R_{-} E_{\text {res }(j)}=\frac{E_{\text {res }(j)}}{E_{\text {ini }(j)}}
$$

Where $E_{\text {ini }(j)}$ and $E_{\text {res }(j)}$ are the initial and residual energy levels of the $j$-th node.

We note that depending on the value of the parameter $\alpha \in[0,1]$ in Eqn.(14) we can determine the weight $w_{s}(i)$ value. For example, a large value of $\alpha$ would give more importance to how much residual energy is left in the node whereas a larger value would give more importance to how much energy has the node already spent.

After calculating the weight of the sensor node, the sensor nodes transmit these data to all other sensor nodes in the cluster. In the next step, all sensor nodes use the received data to calculate the mean weight within their cluster radius as

$$
w_{c}(i)=\sum_{j \in I_{i}} w_{s}(j)
$$

where, $\left|I_{i}\right|$ is the cardinality of the set $I_{i}$.

We then propose modifying the $\mathrm{CH}$ selection probability associated with the $i$-th sensor to be as follows

$$
p_{s i}=p_{s} \frac{w_{s}(i)}{w_{c}(i)}
$$

Then in our EDAC protocol, we apply a similar approach as in other probabilistic clustering methods where the $i$-th node calculates a threshold $T(i)$ given as 


$$
T(i)= \begin{cases}\frac{p_{s i}}{1-p_{s i}\left(\bmod \frac{1}{p_{s i}}\right)}, & i \in G \\ 0 & \text { else }\end{cases}
$$

where, $G$ denotes the set of eligible cluster heads. The $i$-th node then generates a random number between $[0,1]$ and compares the generated number against $T(i)$. If the number is less than $(i)$, then the node becomes a cluster head. The steps of our proposed algorithm are explained in Table 1.

TABLE I. The Proposed Clustering PRotocol (EDAC)

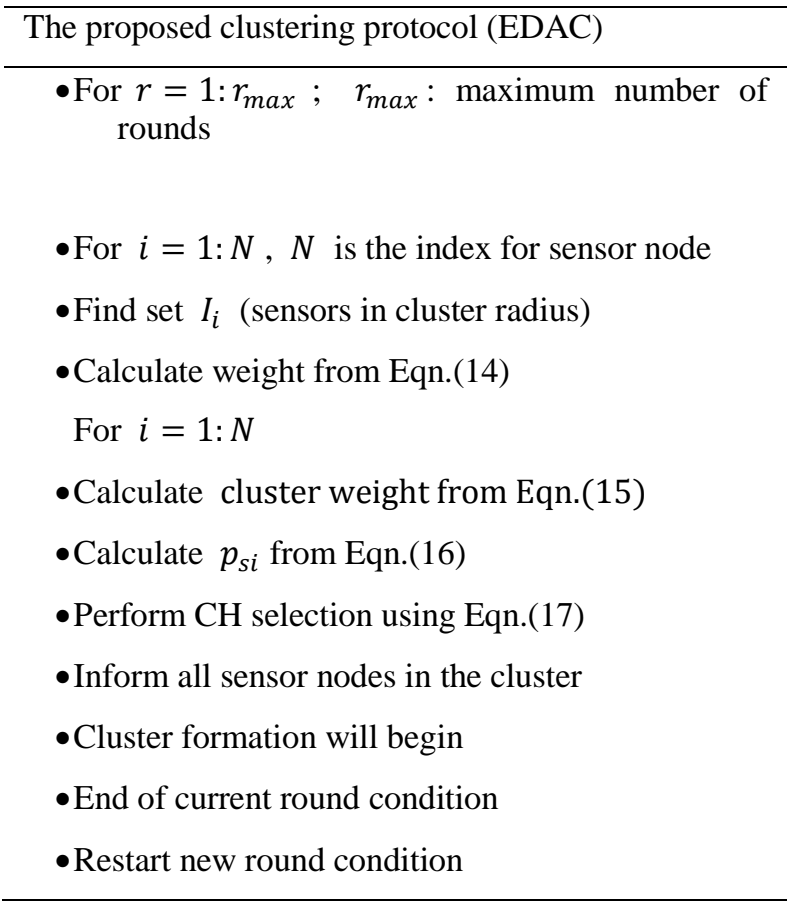

\section{Simulation Results}

In the following experiments, we compare the performance of our proposed algorithm versus that of both the LEACH and DEEC algorithms with emphasis on the network lifetime.

We consider a wireless sensor network with $N=100$ sensor nodes randomly distributed in an area with dimensions of $100 \mathrm{~m} \times 100 \mathrm{~m}$. We assume the BS is in the center of the sensing region. Table (2) summarizes the radio parameters used in simulations. The proposed protocol is compared with LEACH and DEEC protocols.

TABLE II. PARAMETERS USED SimUlations

\begin{tabular}{cl}
\hline \hline Parameter & \multicolumn{1}{c}{ value } \\
\hline$E_{\text {elec }}$ & $500 \mathrm{~nJ} / \mathrm{bit}$ \\
$\epsilon_{f s}$ & $10 \mathrm{PJ} / \mathrm{bit} / \mathrm{m}_{2}$ \\
$\epsilon_{m p}$ & $0.0013 \mathrm{PJ} / \mathrm{bit} / \mathrm{m}_{4}$ \\
$E_{0}$ & $0.5 \mathrm{~J}$ \\
$d_{0}$ & $70 \mathrm{~m}$ \\
\hline
\end{tabular}

\begin{tabular}{rl}
\hline Message size & 4000 bit \\
$p$ & 0.1
\end{tabular}

In the first experiment, we consider a two level heterogeneous network with normal nodes having an initial energy level of $E_{0}$ and advanced nodes having an energy level of $2 E_{0}$. We note that we set $\alpha=0.5$ in our proposed algorithm. We set the percentage of advanced nodes to 0.3. Results are depicted in Fig.(3) and (4) below.

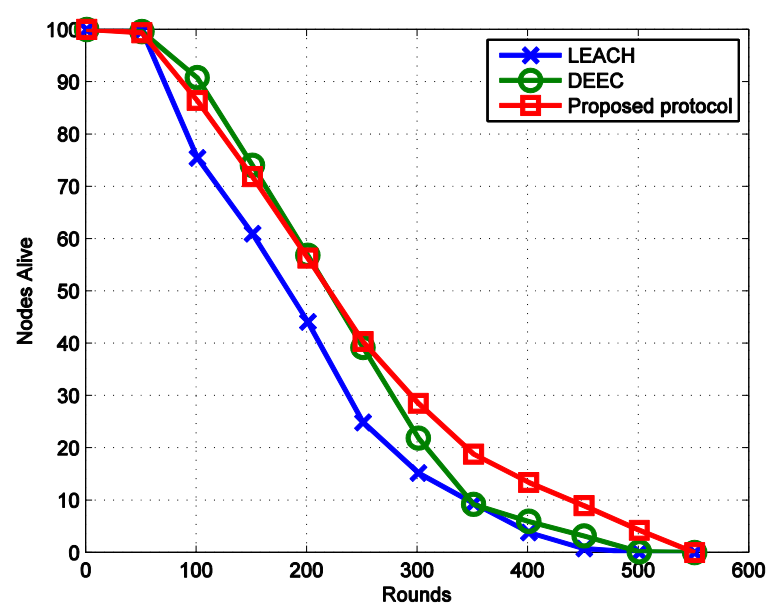

Fig. 3. Alive nodes performance of the LEACH, DEEC and EDAC proposed protocol for a two-level heterogeneous network

From Fig.(3), we can observe the differences in performance between protocols which comes from the different metrics that are used by these protocols in the process of $\mathrm{CH}$ selection. It is clear that the stable time of the proposed protocol is comparable to that of the LEACH and DEEC protocols. Both of DEEC and the proposed protocol achieve higher performance than LEACH protocol. Also, we can note that the unstable region of our proposed protocol is larger than that of LEACH and DEEC protocols, which means more rounds and longer network lifetime. The first sensor node dies after approximately 60 rounds, we expect that this sensor node is a normal node; because the probability of a normal node to die is greater than that of the advanced node. Furthermore, we expect that during the last rounds only the advanced nodes will be alive.

Figure (4) shows that the messages delivered by our proposed protocol are more than that of LEACH and DEEC protocols, the comparison between protocols is made with $\alpha=0.5$. So, our proposed protocol achieves a better throughput. This is a result of the improvement in network lifetime.

We next investigate the performance of our EDAC protocol for two-level heterogeneous networks but for different percentages of advanced nodes (i.e., varying $m$ ). With parameters are the same as used in Table.(2), number of alive nodes and sent packets are depicted in Figs.(5) and (6). One notes that as the number of advanced nodes increases, so does the network's lifetime as evident in Fig.(6). 


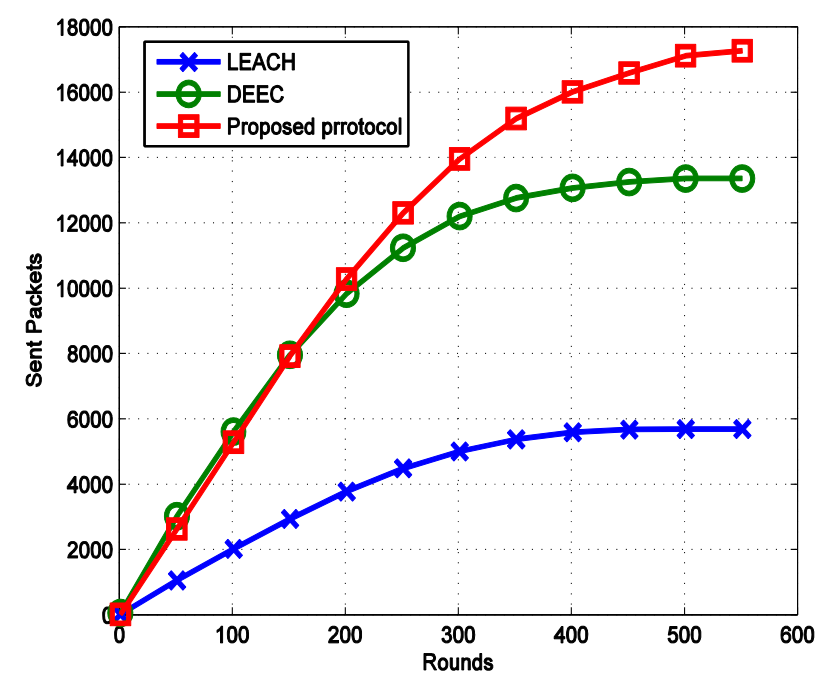

Fig. 4. Number of sent packets for LEACH, DEEC and the proposed protocol

As a result, the accumulative number of sent packets increases as $m$ is increased. This agrees with what we expect from an energy efficient clustering method.

We next investigate the performance of our proposed protocol versus that of the LEACH and DEEC protocols in terms of the network lifetime as we vary the range of initial energies. More precisely, sensors are equipped with random initial energy levels that fall in the range $\left[E_{0}, E_{0}(1+a)\right]$. Thus, we have in effect a multilevel network. Results in Fig.(7) show the first round when $10 \%$ of the nodes die (i.e., consumed their energy) as we vary $a$. It is noted that both the DEEC and our proposed EDAC protocol have a comparable

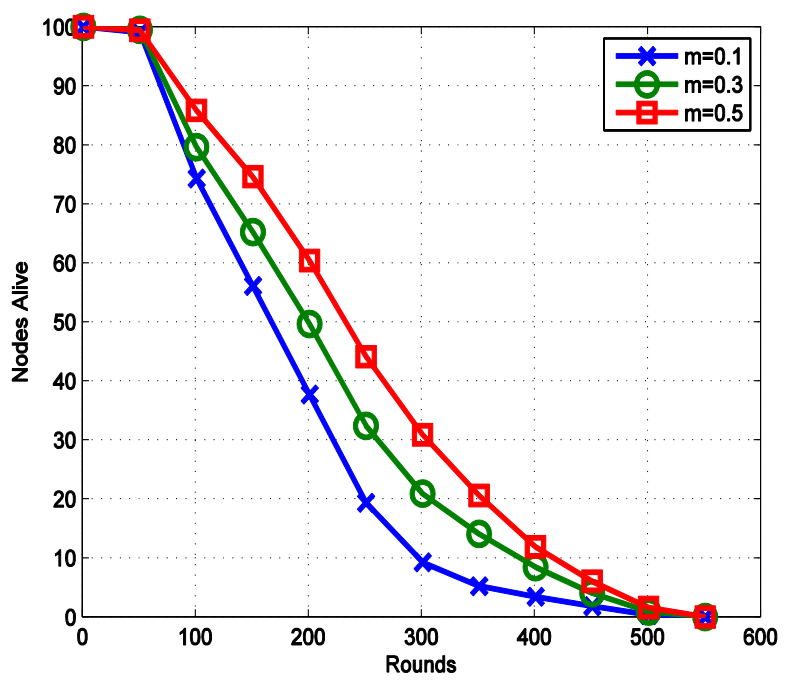

Fig. 5. Number of alive nodes using the EDAC algorithm for various $\boldsymbol{m}$ values

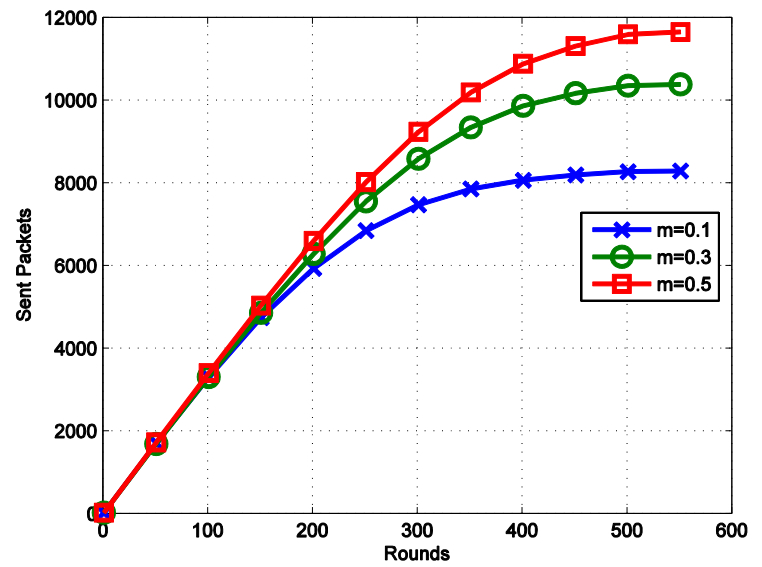

Fig. 6. Number of sent packets using the EDAC algorithm for various $\boldsymbol{m}$ values

performance and they both outperform the LEACH algorithm. On the other hand, Fig. (8) depicts the first round when all sensors have died out. Results show that our proposed method outperforms both the LEACH and DEEC algorithms. In particular, the proposed EDAC extend the network's lifetime by almost $11 \%$ and $37 \%$ with comparison to the DEEC and LEACH protocols, respectively.

\section{CONCLUSION}

In this paper, we propose a probabilistic energy aware clustering algorithm which we call the EDAC. In addition to a sensor's residual energy, the sensor incorporates the residual energy levels of sensors within the cluster radius in addition to the distances between sensors. Using this metric allows selection of cluster head so that it can be close to sensors with varying levels of residual energies (e.g., close to sensors with low residual energy levels). The weighted metric is used in constructing a cluster head selection probability for each sensor. Simulation results, indicate that the proposed algorithm is applicable for both two-level and multi-level networks. In addition, the EDAC performance in terms of network lifetime and sent packets has been shown to outperform both those of the LEACH and DEEC protocols under different setups.

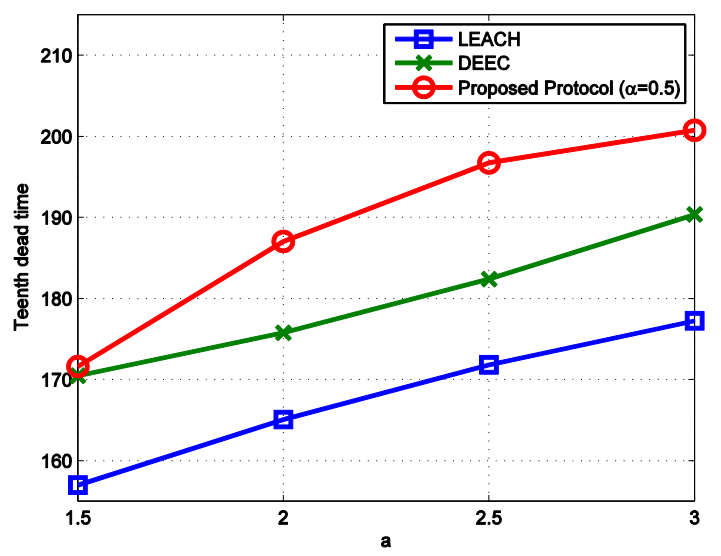

Fig. 7. Round which the time when the first $10 \%$ of the nodes die for different energy ranges 


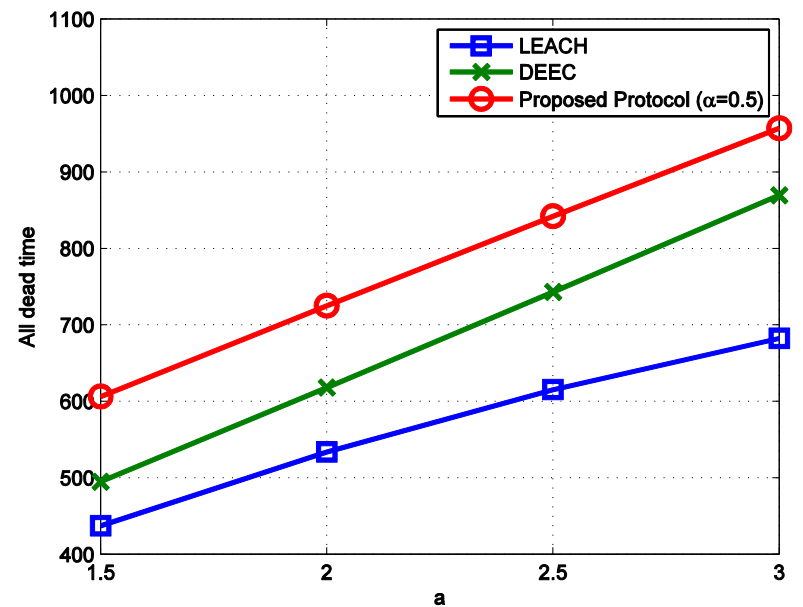

Fig. 8. Round all nodes die for different energy ranges

\section{REFERENCES}

[1] Chong, Chee-Yee and Kumar, Srikanta P, Sensor Networks: Evolution, Opportunities, and Challenges, Proceedings of the IEEE,VOL. 91, NO. 8, (2003), 1247-1256.

[2] M. P. urii; Z. Tafa ; G. Dimi; V. Milutinovi, A Survey of Military Applications of Wireless Sensor Networks, Embedded Computing (MECO), 2012 Mediterranean Conference on,(2011), 196-199.

[3] L. M. Oliveira and J. J. Rodrigues, Wireless Sensor Networks: A Survey on Environmental Monitoring, Journal of communications, VOL. 6, NO. 2,(2003), 143-151.

[4] Garc'ia-Hern'andez, Carlos $\mathrm{F}$ and Ibarguengoytia-Gonzalez, Pablo $\mathrm{H}$ and Garc'ia-Hern'andez, Joaqu'in and P'erez-D'iaz, Jes'us A, Wireless Sensor Networks and Applications: A Survey, IJCSNS International Journal of Computer Science and Network Security,VOL. 7, NO. 3,(2007), 264- 273.
[5] Arampatzis, Th and Lygeros, John and Manesis, Stamatis, A Survey of Applications of Wireless Sensors And Wireless Sensor Networks, Intellegent Control, 2005. Proceedings of the 2005 IEEE International Sympsium on, Mediterrean Conference on Control and Automation,(2005), 719-724.

[6] Liu, Xuxun, A Survey on Clustering Routing Protocols inWireless Sensor Networks, Sensors,VOL. 12, NO. 8,(2012), 11113-11153.

[7] Heinzelman, Wendi Rabiner and Chandrakasan, Anantha and Balakrishnan, Hari, Energy-Efficient Communication Protocol for Wireless Microsensor Networks, System sciences, 2000. Proceedings of the $33^{\text {rd }}$ annual Hawaii international conference on, IEEE,VOL. 12, NO. $8,(2000), 10-$ pp

[8] Younis, Ossama and Fahmy, Sonia, HEED: A Hybrid, Energy-Efficient, Distributed Clustering Approach for Ad Hoc Sensor Networks, Mobile Computing, IEEE Transactions on,VOL. 3, NO. 4,(2004), 366-379

[9] Qing, Li and Zhu, Qingxin and Wang, Mingwen, Design of a Distributed Energy-Efficient Clustering Algorithm for Heterogeneous Wireless Sensor Networks, Computer communications, VOL. 29, NO. 12,(2006), 2230- 2237

[10] Smaragdakis, Georgios and Matta, Ibrahim and Bestavros, Azer and others, SEP: A Stable Election Protocol for Clustered Heterogeneous Wireless Sensor Networks, Second international workshop on sensor and actor network protocols and applications (SANPA 2004),VOL. 3, NO. 8,(2004), 11113-11153.

[11] Bandyopadhyay, Seema and Coyle, Edward J, An Energy Efficient Hierarchical Clustering Algorithm for Wireless Sensor Networks, INFOCOM 2003. Twenty-Second Annual Joint Conference of the IEEE Computer and Communications. IEEE Societies,VOL. 3, NO. 8,(2003), 1713-1723.

[12] Abo-Zahhad, Mohammed and Ahmed, Sabah M and Sabor, Nabil and Sasaki, Shigenobu, A New Energy-Efficient Adaptive Clustering Protocol Based on Genetic Algorithm for Improving the Lifetime and the Stable Period of Wireless Sensor Networks, International Journal of Energy Information and Communications,VOL. 5, NO. 3,(2014).

[13] Heinzelman, Wendi B and Chandrakasan, Anantha P and Balakrishnan Hari, An Application-Specific Protocol Architecture for Wireless Microsensor Networks, Wireless Communications, IEEE Transactions on,VOL. 1, NO. 4,(2002), 660-670. 